\title{
Morphology-dependent space charge polarization and dielectric relaxation of $\mathrm{CdO}$ nanomorphotypes
}

\author{
Paulose Thomas and K. E. Abraham* \\ Department of Physics, St. Berchmans College \\ Changanassery, 686 101, Kerala, India \\ *abrahamke@gmail.com
}

Received 8 August 2016; Revised 31 October 2016; Accepted 1 November 2016; Published 12 December 2016

\begin{abstract}
A versatile approach signifying the morphology-dependent dielectric polarization and relaxation mechanisms of cadmium oxide $(\mathrm{CdO})$ nanosphere, nanoflakes and nanoparallelepiped morphotypes as a function of frequency and temperature is presented. Variation of dielectric property is observed due to the changes of space charge/interfacial polarization resulting from the variations of surface to volume ratio of nanomorphology. Accordingly, colossal dielectric constant value has been observed in CdO nanosphere having larger surface to volume ratio. The order of dielectric constant $(\mathrm{dc})$ values observed for the present nanomorphologies is: $\mathrm{dc}$ of sphere $>\mathrm{dc}$ of flakes $>\mathrm{dc}$ of parallelepiped resembles the order of surface to volume ratios of the present morphologies respectively. The experimental data of complex impedance values are numerically fitted using theoretical models which provide the information of role of grain resistance on dielectric polarization and Cole-Cole type mechanism of dielectric relaxation process. The activation energies for electron transport are found to be $0.087 \mathrm{eV}$ for spheres, $0.074 \mathrm{eV}$ for flakes and 0.067 for parallelepiped nanomorphotypes of $\mathrm{CdO}$. The dielectric and impedance spectroscopic analysis of the present material opens up wide scope for morphology-dependent tuning of nanomaterials for electrical applications.
\end{abstract}

Keywords: $\mathrm{CdO}$ nanomorphotype; space charge polarization; impedance spectroscopy; Cole-Cole dielectric relaxation.

\section{Introduction}

In the recent years dielectric properties of metal oxide semiconductor nanomaterials have caught additional attention because of its high dielectric constant values at nanosize range. The large surfaces to volume ratio and quantum confinement effect have altered dielectric and conductivity behavior of nanomaterials much compared with its bulk materials. In addition, researchers generally believe that the morphology of nanomaterials is an important parameter for modifying the entire physical properties like optical, electrical, magnetic and nonlinear behavior. Over the past decades many researchers have satisfactorily examined electrical transport and dielectric behavior of semiconductor metal oxide nanomaterials with various parameters including temperature, particle size, structural defects, effect of $\mathrm{pH}$, doping, etc. Liao et al. presented the surface state effect on electron transport in individual $\mathrm{ZnO}$ nanowires and confirm that the surface states have greatly affected the electronic transport in single $\mathrm{ZnO}$ nanowires. ${ }^{1}$ Dang and co-workers examined the dielectric properties and morphologies of composites filled with whisker and nanosized $\mathrm{ZnO}^{2}$ They claim that dielectric constant and losses increase with increasing w-ZnO content in composites. Suresh conducted the study of dielectric relaxation behavior of CdS nanoparticles and found that dielectric properties of CdS nanoparticles significantly enhanced specially in the low frequency range due to confinement. ${ }^{3}$ Velayutham et al. presented the theoretical and experimental approach on dielectric properties of $\mathrm{ZnO}$ nanoparticles and polyurethane/ZnO nanocomposites. ${ }^{4}$ El-Sayed and co-workers carried out a study on synthesis, characterization, optical and dielectric properties of polyvinyl chloride/Cadmium oxide (CdO) nanocomposite films. ${ }^{5}$ They concluded that optical and dielectric properties are reinforced by adding the CdO nanoparticles in PVC matrix. Tripathi et al. studied the dielectric relaxation and ac conductivity mechanism of $\mathrm{CdO}$ nanoparticles recently and suggests that the electron transport mechanism obeys the universal power law. ${ }^{31}$ Out of these important literatures, we observe that very little studies are carried out to dig out the dielectric property of $\mathrm{CdO}$ semiconductor nanomaterial.

$\mathrm{CdO}$ is an n-type IIB-VIA, semiconductor metal oxide with direct bandgap of $2.5 \mathrm{eV}$ and indirect bandgap of $1.98 \mathrm{eV}^{6,7}$ Recently many researchers have shown interest on synthesis of $\mathrm{CdO}$ nanomaterials especially in diverse morphotypes, because of its potential applications in electronic storage devices, optoelectronics, gas sensing, solar cells and flat panel displays. ${ }^{8-11}$ However, no report is observed on the study of dielectric properties of nanostructures as a function of different morphologies. In the present study, electrical property of three different morphologies of $\mathrm{CdO}$ (sphere, flakes and parallelepiped) nanostructures are compared and concluded that the morphology is a parameter that have control over electrical properties of materials. In addition to this we have employed the impedance spectroscopy to determine the

This is an Open Access article published by World Scientific Publishing Company. It is distributed under the terms of the Creative Commons Attribution 4.0 (CC-BY) License. Further distribution of this work is permitted, provided the original work is properly cited. 
electrical resistance of electrode, grain and grain boundary and confirm which of them is responsible for dielectric polarization process and also analyze the dielectric relaxation mechanism. To the best of our understanding this is the first ever made report to study the dependence of dielectric property of $\mathrm{CdO}$ nanostructure on surface morphologies.

\section{Experimental Procedure}

\subsection{Synthesis of CdO nanomorphotypes}

We have already reported the chemical synthesis route and growth mechanism for fabricating $\mathrm{CdO}$ nanomorphotypes through chemical precipitation and hydrothermal method using $\left(\mathrm{CH}_{3} \mathrm{COO}\right) \mathrm{Cd} \cdot 2 \mathrm{H}_{2} \mathrm{O}$ and $\mathrm{NaOH}$ as starting materials. ${ }^{14}$ In brief, the chemical precipitation method is used for synthesizing the spherical nanomorphotype. The solutions of reactants in double distilled water are prepared in 1:1 ratio. $\mathrm{NaOH}$ solution is added to $\left(\mathrm{CH}_{3} \mathrm{COO}\right) \mathrm{Cd} \cdot 2 \mathrm{H}_{2} \mathrm{O}$ solution dropwise with constant stirring using a magnetic stirrer for 30 min. By the addition of $\mathrm{NaOH}$, a milky turbid solution is formed due to the precipitation of $\mathrm{Cd}(\mathrm{OH})_{2}$ spherical precursor nanostructure. The flake and parallelepiped morphotypes have been prepared by hydrothermal method. A specific amount of capping agent $\mathrm{C}_{6} \mathrm{H}_{15} \mathrm{NO}_{3}$ (TEA), is added ( $1 \mathrm{~mL}$ per $100 \mathrm{~mL}$ solvent) to the same precursor solution and the mixture is poured into a Teflon lined stainless steel autoclave. It is then heated at $200^{\circ} \mathrm{C}$ for 8 and $24 \mathrm{~h}$ for the formation of $\mathrm{Cd}(\mathrm{OH})_{2}$ nanoflakes and $\mathrm{Cd}(\mathrm{OH})_{2}$ parallelepiped precursor nanostructures, respectively. At the end of chemical precipitation and hydrothermal treatment, white precursors are obtained which are then dried at $60^{\circ} \mathrm{C}$ for $4 \mathrm{~h}$. The yellowish colored $\mathrm{CdO}$ nanomorphotypes are obtained by annealing the precursors at $500^{\circ} \mathrm{C}$ for $5 \mathrm{~h}$ in air. The variation of morphologies at various stages of synthesis is shown with the help of transmission electron microscope images presented in Fig. 1.

\subsection{Analysis techniques}

The crystal structure (X-ray diffraction analysis- Bruker AXS D8 Advance with X-ray source $\mathrm{Cu} \mathrm{Ka}$, Wavelength $1.5406 \AA$ A), BET surface area analysis (Micromeritics Gemini 2375 V5.01, USA), Sample morphologies and size dimensions (high resolution transmission electron microscopeJEM-2100, $200 \mathrm{Kv}$, Jeol) and the fundamental optical bandgap (optical reflection spectroscopy-T90+UV/Vis spectrometer) are already reported in our recent publication. ${ }^{14}$ For electrical analysis, the prepared powder samples are pelletized at a pressure of 2.5 tons for $5 \mathrm{~min}$ using a $\mathrm{KBr}$ hydraulic press. The pellet is then sintered at temperature of $400^{\circ} \mathrm{C}$ for $10 \mathrm{~h}$ for densification to avoid the voids/air traps between the grains/grain boundaries. The disc shaped pellet has a radius of $0.65 \mathrm{~cm}$ and thickness of $0.18 \mathrm{~cm}$. The density of sintered pellet is $8.49 \mathrm{~g} / \mathrm{cm}^{3}$ which is much closer to the density $8.15 \mathrm{~g} / \mathrm{cm}^{3}$ of crystalline $\mathrm{CdO}$ material. When the pellets are prepared by pressing powdered sample using hydraulic press, there are chances to form many voids or air gaps within the pellet. Such air gaps can affect the measurements of electrical/dielectric properties of the material. The sintering process reduces the air gaps or voids inside the pellet and the pellet becomes highly dense. The higher density of the pellet is due to sintering and close packing of nanoparticles. ${ }^{32,33}$ Silver paste is coated on both sides of the pellet for creating better contact between the pellet and the electrodes. The pellets are placed in a furnace for determining the effect of dielectric property on temperatures. The dielectric and impedance spectrum measurements are carried out using HIOKI 3532-50 LCR HiTESTER instrument having frequency range $50 \mathrm{~Hz}$ to $5 \mathrm{MHz}$.

The XRD spectra ${ }^{14}$ given in Fig. 2(I) shows that the diffraction peaks of the samples are very sharp and highly intense which reveals the well-defined crystalline nature and phase purity of the prepared nanostructures. The diffraction pattern of CdO at (111), (200), (220), (311) and (222) planes

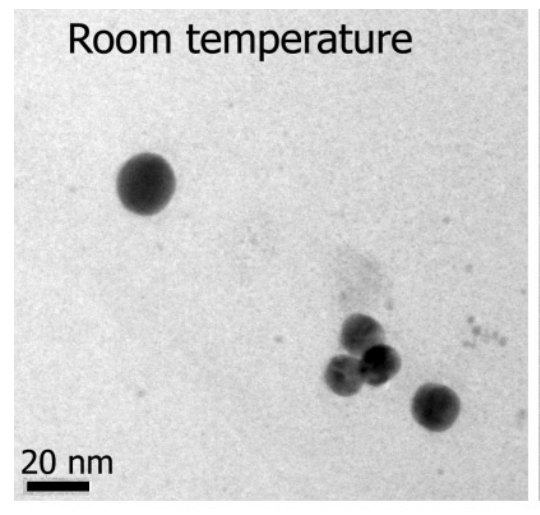

(a)

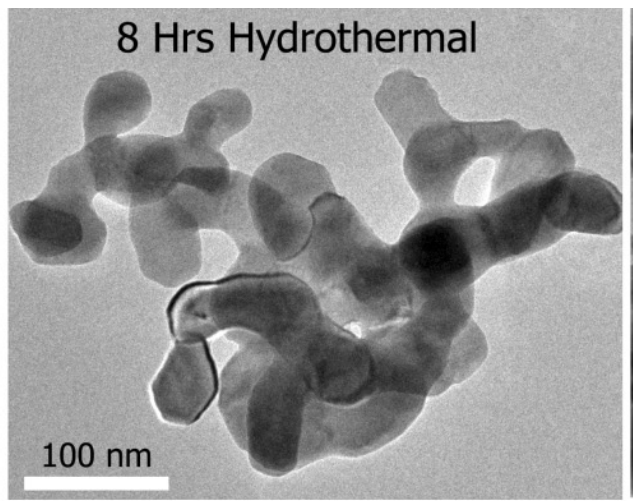

(b)

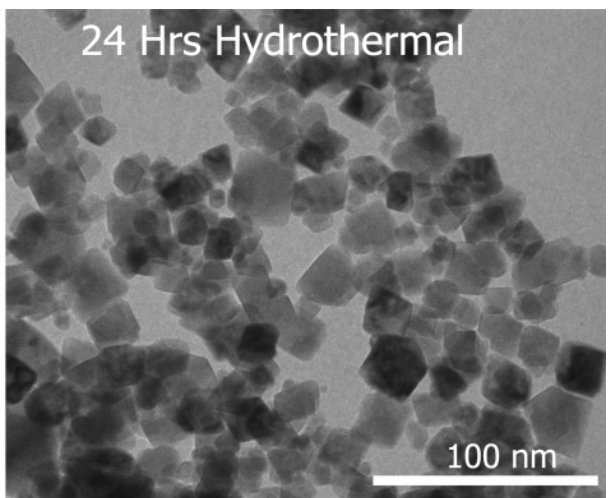

(c)

Fig. 1. Transmission electron microscope images of $\mathrm{CdO}$ nanomorphotypes annealed at $500^{\circ} \mathrm{C}$ for $5 \mathrm{~h}$ in air: (a) nanosphere, (b) nanoflakes and (c) nanoparallelepiped morphologies, respectively. 

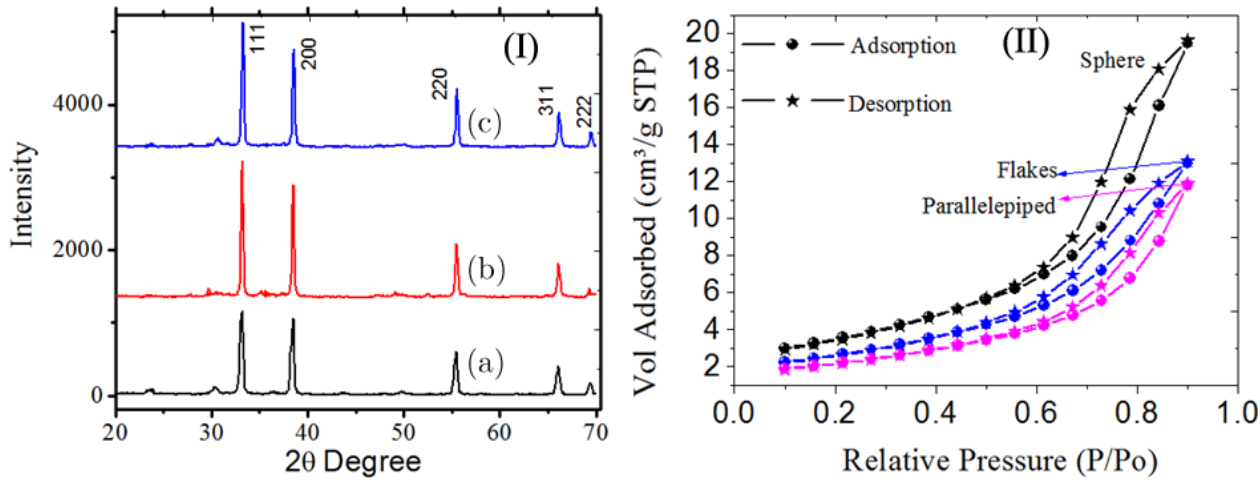

Fig. 2. (I) XRD pattern of CdO: (a) sphere, (b) flakes, (c) parallelepiped. (II) BET (isothermal curve) surface area analysis of CdO nanomorphotype.

Table 1. Parameters obtained from BET surface area analysis.

\begin{tabular}{lccc}
\hline & Sphere & Flakes & Parallelepiped \\
\hline Single point surface area $\left(\mathrm{m}^{2} / \mathrm{g}\right)$ & 12.3464 & 9.2851 & 7.6702 \\
BET surface area $\left(\mathrm{m}^{2} / \mathrm{g}\right)$ & 12.5955 & 9.5109 & 7.7606 \\
Langmuir surface $\mathrm{area}\left(\mathrm{m}^{2} / \mathrm{g}\right)$ & 20.4812 & 15.5189 & 12.5074 \\
Micropore area $\left(\mathrm{m}^{2} / \mathrm{g}\right)$ & 0.6818 & 0.2945 & 0.8439 \\
External surface $\operatorname{area}\left(\mathrm{m}^{2} / \mathrm{g}\right)$ & 11.9137 & 9.2165 & 6.9167 \\
Micropore volume $\left(\mathrm{cm}^{3} / \mathrm{g}\right)$ & 0.000233 & 0.000055 & 0.000380 \\
BJH adsorption average pore & 74.0469 & 67.1888 & 76.9408 \\
$\quad$ & & & \\
\hline
\end{tabular}

match well with the JCPDS card No. 05-0640 of CdO having lattice constant $a=4.695 \AA$. The BET spectra ${ }^{14}$ given in Fig. 2(II) indicates that the surface area is maximum in the case of spherical shape and that the surface area of flakes structure is in between spherical and parallelepiped nanostructures. The surface area measurements ${ }^{14}$ are given in Table 1. The BET surface area analysis of the materials confirm the morphology-dependent variation of nanostructures surface area.

\section{Results and Discussion}

\subsection{Dielectric polarization and relaxation}

A dielectric material is made up of large number of atoms/ molecules that possess different types of electric polarizations such as electronic, atomic/ionic, dipolar, orientational or hopping and space charge polarizations. The space charge, orientational and atomic/ionic polarizations are significant only in low frequency $\mathrm{AC}$ field, whereas electronic polarizations are more significant in high frequency AC field. ${ }^{15}$ Therefore in the present study we analyze the dielectric property of $\mathrm{CdO}$ nanostructure as functions of frequency and temperature. The frequency-dependent dielectric constant and loss factor $(\tan \delta)$ at room temperature and temperaturedependent dielectric constant at $55 \mathrm{~Hz}$ and $100 \mathrm{kHz}$ are represented in Fig. 3. It is noteworthy that the observed dielectric value of $\mathrm{CdO}$ nanosphere is about 6530 whereas the dielectric constant of bulk $\mathrm{CdO}$ is about $21 .{ }^{12}$ As the dielectric constant $\varepsilon_{r}>10^{3}$ is termed as Colossal, ${ }^{13}$ the value of dielectric constant obtained for $\mathrm{CdO}$ spherical nanomorphotype can be termed as colossal. The colossal value of room temperature dielectric constant of $\mathrm{CdO}$ nanostructures has never been reported earlier. Meanwhile, for each morphology, $\tan \delta$ exhibit clear loss peaks corresponding to the frequency at which dielectric constant is maximum. The larger value of dielectric constant and loss factor in $\mathrm{CdO}$ spherical morphology is due to the larger surface to volume ratio of the spherical shapes. In the present study, we are attempting to elucidate the dependency of dielectric polarization and relaxation of $\mathrm{CdO}$ nanostructures on the surface morphology based on dielectric measurement and BET surface area analysis (Fig. 2(II)). ${ }^{14}$ We have also reported on theoretical and experimental grounds that spherical nanomorphology have maximum surface to volume ratio compared to that of $\mathrm{CdO}$ flakes and parallelepiped morphology. ${ }^{14}$ In addition to this we have evidently proved that the size dimension of $\mathrm{CdO}$ nanomorphotypes increases as morphology changes from spherical to flakes and to parallelepiped shape whereas, the surface to volume ratios decreases with increase in particle size dimensions. ${ }^{14}$ The exact reason for change in the dielectric constant behavior with morphology is not clearly understood. However, we explain the dependency of morphology on dielectric behavior of nanomaterials on the basis of particle size dimension as well as surface to volume ratios. For a spherical nanostructure, the general relation for the ratio between surface area to volume $(S / V)$ is $3 / R$ (i.e., $3: R$ ) and for parallelepiped structure, it is $6 / L$ (i.e., $6: L$ ) where $R$ and $L$ are particle size dimensions for spherical and parallelepiped nanostructures, respectively. In the present case nanosphere has lesser size than the remaining morphologies. ${ }^{14}$ Hence, the nanospheres possess maximum surface to volume ratio. Since nanoflakes are similar to parallelepiped shape with one of the dimensions very small, their surface to volume ratio will lie between that of nanosphere and nanoparallelepiped structures. BET analysis also confirms that the surface to volume ratio of flakes is in between that of sphere and parallelepiped nanostructures. Because of small size of 

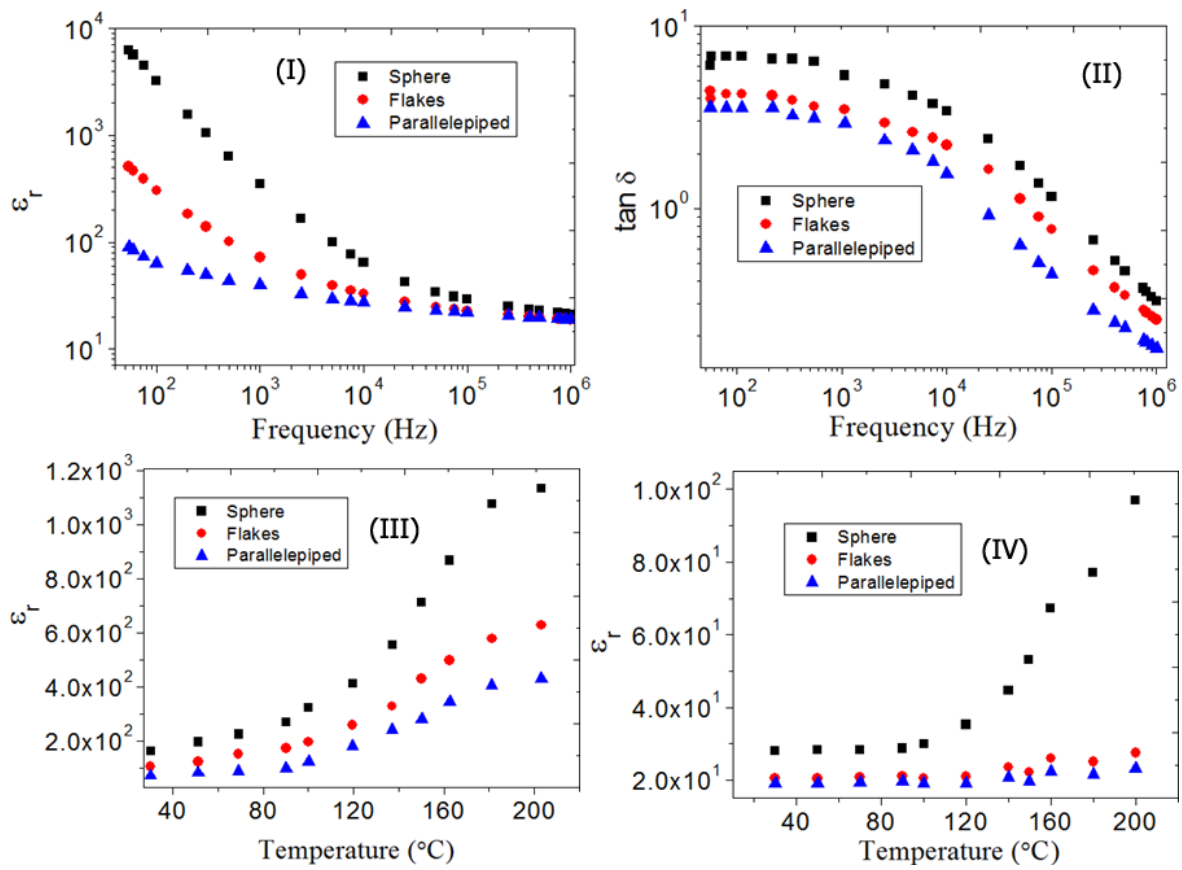

Fig. 3. (I) and (II) Frequency-dependent dielectric constant $\left(\varepsilon_{r}\right)$ and loss factor $(\tan \delta)$ respectively at room temperature. (III) and (IV) Temperature-dependent dielectric constant at $55 \mathrm{~Hz}$ and $100 \mathrm{kHz}$, respectively.

the nanoparticles, more charge carriers reach the surface of the particles. ${ }^{34-36}$ Accordingly, due to the higher surface to volume ratio, there is maximum density of the distribution of charges on the surface of spherical nanostructures. Hence, stronger size selective surface charge effect is possible in spherical morphotypes than that of flake and parallelepiped structures. Consequently, the higher surface charge density creates higher surface charge polarization in the nanosphere compared to other cases. Hence, dielectric constant of nanosphere must be larger than the other morphologies of the present material. It is evident from the measurements that dielectric constant of parallelepiped morphotype shows almost same nature as that of dielectric value of $\mathrm{CdO}$ bulk material whereas the dielectric constant of flakes appears in between that of sphere and parallelepiped nanostructure. Similar morphology-dependent optical, surface plasmons, catalytic activity and electrical behaviors are seen in literatures. ${ }^{37-39}$

The dielectric constant of $\mathrm{CdO}$ morphotypes as a function of temperature at $55 \mathrm{~Hz}$ and $100 \mathrm{kHz}$ are shown in Figs. 3(III) and 3(IV), respectively. In the case of all morphologies the dielectric constant value increases with temperature. The increment of dielectric constant with increase in temperature is due to the temperature-dependent dielectric polarization of nanoparticles. In all these results the dielectric constant of spherical $\mathrm{CdO}$ morphotype has relatively higher value compared to other morphotypes. The order of the dielectric constants obtained is: dielectric constant (d.c.) of nanosphere $>$ d.c. of nanoflake $>$ d.c. of nanoparallelepiped. Hence it could be robustly concluded that the dielectric constant of $\mathrm{CdO}$ nanostructures are significantly dependent on the morphology. The morphology-dependent dielectric analysis in the present study strongly agrees to our previous report on morphology-dependent nonlinear optical behavior of $\mathrm{CdO}$ nanomorphotype ${ }^{14}$ Moreover based on the observed dielectric constant values, the $\mathrm{CdO}$ spherical morphology is a good candidate for using in the energy storage devices.

\subsection{The complex impedance spectrum}

The impedance spectroscopy shows the frequency dependence of real part $\left(Z^{I}\right)$ and imaginary part $\left(Z^{I I}\right)$ of the impedance. The complex impedance spectrum of the present study helps us to reconfirm the picture of morphologydependent dielectric behavior of the present material. It shows the dielectric polarization/relaxation mechanism and contribution of electrode, grain or grain boundary resistance to the dielectric property of $\mathrm{CdO}$ nanomorphotypes. ${ }^{16-18}$ Generally, the dielectric polarizations can be grouped into two: the polarization in resonance region and the polarization in relaxation region. ${ }^{19}$ The polarization associated with vibration of electrons or vibration of atoms/ions belongs to resonance region because resonances will occur when the frequency of excited field is close to the natural frequency of oscillation system. Polarizations by orientational polarization or hopping and space charge polarization belong to the relaxation region. The relaxation phenomena occur only when the time required for the charge carriers to overcome the inertia is available. The Cole-Cole $\left(Z^{I}\right.$ versus $Z^{I I}$ with frequency) plot of $\mathrm{CdO}$ nanosphere, nanoflakes and nanoparallelepiped morphologies at $30^{\circ} \mathrm{C}, 100^{\circ} \mathrm{C}, 150^{\circ} \mathrm{C}$ and $200^{\circ} \mathrm{C}$ are given in Figs. 4(a)-4(c), respectively. The impedance 


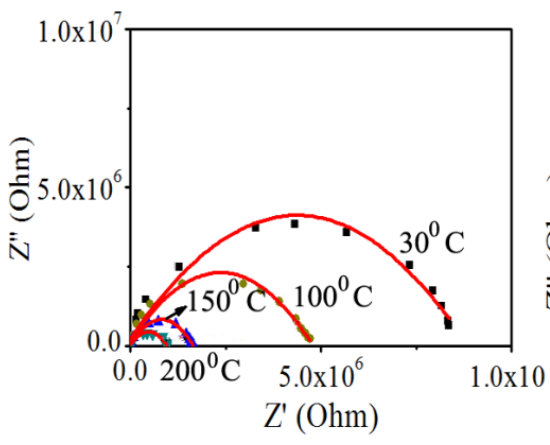

(a)

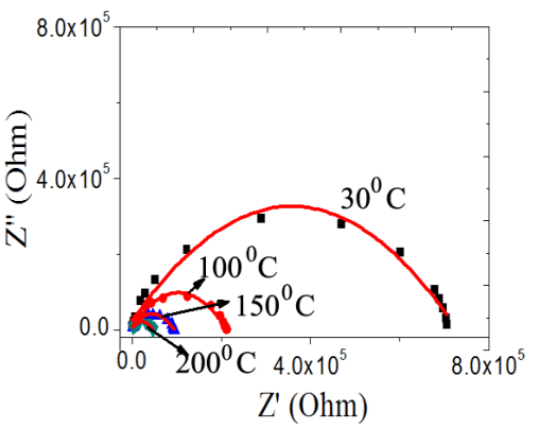

(c)

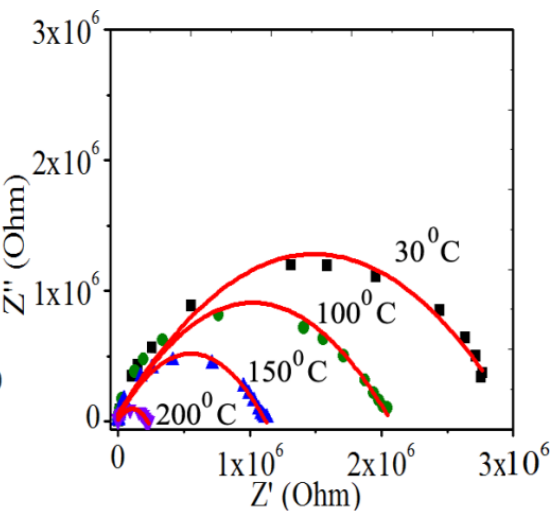

(b)

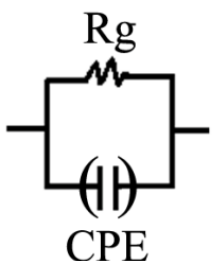

(d)

Fig. 4. The complex impedance spectrum: (a) nanosphere, (b) nanoflakes and (c) nanoparallelepiped shapes, respectively. (d) R-CPE equivalent circuit. (The dotted line indicates experimental data points and solid line indicates numerical fitting to the experimental data point by equivalent circuit).

analysis of crystalline materials can be explained by fitting the experimental data of impedance value to an equivalent circuit model consisting of a parallel combination of an ohmic resistor $R$ and a capacitor $C$. The Nyquist (Cole-Cole plot) diagram of such a circuit gives one to three semicircular plots. Usually the diameter of low frequency side arc (high $Z^{I}$ ) represents the electrode resistance $(R)$. The next arc gives the grain resistance and the high frequency side arc gives the grain boundary resistance. However, in some cases the experimental data show a deviation from this simple $R-C$ behavior and the diagram need not be a semicircle plot. It may look like a deformed, broadened or depressed semicircle. In such cases, we can generally use parallel R-CPE equivalent circuit instead of parallel $R-C$ circuit. $^{20,21}$

For the present impedance spectra, the experimental data are fitted to an equivalent circuit consisting of a resistor and a constant phase element (CPE) instead of a capacitor having capacitance $C$. The proposed equivalent circuit used for simulating the experimental data in the present work is shown in Fig. 4(d) ${ }^{26}$ Here the total impedance is given by the relation $^{23-26}$

$$
Z^{*}=\left(\frac{1}{R}+\frac{1}{Z_{\mathrm{CPE}}}\right)^{-1}
$$

where $Z_{C P E}=Q(i \omega)^{n}, i$ is the imaginary unit, $Q$ is the prefactor for $\mathrm{CPE}$ and $n$ is its exponent. The exponent $n$ of $\mathrm{CPE}$ varies from 0 to $1 . n=1$ defines the impedance of ideal capacitor. $n<1$ shows the departure from the ideal capacitor behavior, commonly known as Cole-Cole type relaxation behavior of dielectric materials.

$$
Z^{\prime}=\frac{R\left(1+R Q \omega^{n} \cos (n \pi / 2)\right)}{1+2 R Q \omega^{n} \cos (n \pi / 2)+\left(R Q \omega^{n}\right)^{2}}
$$

and

$$
Z^{\prime \prime}=-\frac{R^{2} Q \omega^{n} \sin (n \pi / 2)}{1+2 R Q \omega^{n} \cos (n \pi / 2)+\left(R Q \omega^{n}\right)^{2}} .
$$

According to the above relations, the real capacitance can be calculated by the relation

$$
C=R^{(1-n / n)} Q^{(1 / n)} .
$$

The impedance spectrum (Figs. 4(a)-4(c)) shows that at all temperatures $Z^{I}$ versus $Z^{I I}$ curve appears like a 'depressed single semicircle', indicating the contribution of grain resistance $R_{g}$ and grain capacitance $C_{g}$ in dielectric polarization of the nanomaterials. In other words, the diameter of these depressed semicircular arcs represents the resistance (in $\mathrm{k} \Omega$ range) contributed by the grain resistance. ${ }^{27,28}$ 
Table 2. The grain parameters of $\mathrm{CdO}$ nanomorphotype by numerical fit to the experimental data points.

\begin{tabular}{lccccc}
\hline Sphere & $R_{g}(\Omega)$ & $C_{g}(F)$ & $\sigma_{g}(S / \mathrm{cm})$ & $\tau_{g}(s)$ & ' $n$ ' values \\
\hline $30^{\circ} \mathrm{C}$ & $9.48 \times 10^{6}$ & $3.93 \times 10^{-11}$ & $9.54 \times 10^{-7}$ & $3.73 \times 10^{-4}$ & 0.78 \\
$100^{\circ} \mathrm{C}$ & $4.84 \times 10^{6}$ & $4.37 \times 10^{-11}$ & $1.87 \times 10^{-6}$ & $2.12 \times 10^{-4}$ & 0.73 \\
$150^{\circ} \mathrm{C}$ & $2.33 \times 10^{6}$ & $4.58 \times 10^{-11}$ & $3.88 \times 10^{-6}$ & $1.07 \times 10^{-4}$ & 0.69 \\
$200^{\circ} \mathrm{C}$ & $1.10 \times 10^{6}$ & $5.89 \times 10^{-11}$ & $8.22 \times 10^{-6}$ & $6.48 \times 10^{-5}$ & 0.63 \\
Flakes & & & & & \\
$30^{\circ} \mathrm{C}$ & $2.99 \times 10^{6}$ & $2.54 \times 10^{-11}$ & $3.03 \times 10^{-6}$ & $7.59 \times 10^{-5}$ & 0.76 \\
$100^{\circ} \mathrm{C}$ & $3.05 \times 10^{6}$ & $2.93 \times 10^{-11}$ & $2.97 \times 10^{-6}$ & $8.94 \times 10^{-5}$ & 0.71 \\
$150^{\circ} \mathrm{C}$ & $1.12 \times 10^{6}$ & $4.69 \times 10^{-11}$ & $8.08 \times 10^{-6}$ & $5.25 \times 10^{-5}$ & 0.68 \\
$200^{\circ} \mathrm{C}$ & $2.39 \times 10^{5}$ & $8.99 \times 10^{-11}$ & $3.78 \times 10^{-5}$ & $2.15 \times 10^{-5}$ & 0.63 \\
Parallelepiped & & & & & \\
$30^{\circ} \mathrm{C}$ & $7.24 \times 10^{5}$ & $2.34 \times 10^{-11}$ & $1.25 \times 10^{-5}$ & $1.69 \times 10^{-5}$ & 0.69 \\
$100^{\circ} \mathrm{C}$ & $2.38 \times 10^{5}$ & $2.48 \times 10^{-11}$ & $3.80 \times 10^{-5}$ & $5.90 \times 10^{-6}$ & 0.64 \\
$150^{\circ} \mathrm{C}$ & $9.58 \times 10^{4}$ & $2.99 \times 10^{-11}$ & $9.44 \times 10^{-5}$ & $2.86 \times 10^{-6}$ & 0.59 \\
$200^{\circ} \mathrm{C}$ & $4.35 \times 10^{4}$ & $3.45 \times 10^{-11}$ & $2.08 \times 10^{-4}$ & $1.50 \times 10^{-6}$ & 0.54 \\
\hline
\end{tabular}

The value of $R_{g}$ is obtained from the diameter of impedance semicircle on $X$-axis. The mean relaxation time is obtained from the relation $\tau_{g}=R g C g,{ }^{21}$ which is the inverse of the most intense frequency $\omega_{g}$ of the impedance spectrum. The electrical parameters of $\mathrm{CdO}$ nanomorphotypes obtained by fitting the experimental data to the equivalent circuit are reported in Table 2. The resistances obtained in all temperatures are due to grain response which also indicates the polycrystalline nature of the prepared material.

It is noticed from Table 2, that the grain interior resistance and relaxation time are decreased with corresponding increment in temperatures. The variations of fitted $n$ values and relaxation time for different temperatures are evidences for Cole-Cole type relaxation mechanism in $\mathrm{CdO}$ nanomorphotypes or the departure from Debye behavior, which is further supported by Fig. 5. In Fig. 5, the relaxation frequency is shifted with respect to increase in temperature which is the sign or evidence of the Cole-Cole type relaxation mechanism in dielectric materials. ${ }^{23}$

The activation energy of $\mathrm{CdO}$ nanomorphotypes are calculated by fitting the data through Arrhenius equation ${ }^{29,30}$

$$
\sigma_{g}=\sigma_{0} e^{\left(E a / k_{\mathrm{B}} T\right)}
$$

where $E_{a}$ is the activation energy for conduction. The activation energy is the energy required for a material to once start the conduction process which mainly depends on temperature, size and shape of the nanomaterial. ${ }^{31}$

In Fig. 6, the conductivity values (corresponding to the temperature-dependent resistance values) are plotted against $1000 / T$. The values of activation energy of grain response for $\mathrm{CdO}$ nanomorphotypes are calculated from the slope of the conductivity versus $1000 / T$ curve and are of the order of $0.087 \mathrm{eV}$ for sphere, $0.074 \mathrm{eV}$ for flakes and $0.067 \mathrm{eV}$ for parallelepiped nanomorphotypes. In the present context, the activation energy can be explained as the minimum energy required for a material to start up the electrical transport mechanism. It is activated by thermal and electrical process. Dielectric semiconductor materials with high activation energy indicate that the material is more resistive against electron transport and also have high dielectric polarization/ dielectric constant value. ${ }^{40-43}$

As the resistance increase, the high dielectric polarization energy in the materials gets dissipated in the form of heat which causes large dielectric loss values in the material. In the present study, the higher activation energy in spherical morphology is due to its large grain resistance and dielectric loss values over flakes and parallelepiped morphotypes. It may be noted that the impedance spectroscopic studies given above show larger grain resistance for spherical morphology. Therefore, the calculated activation energy also supports our claim for morphology-dependent dielectric properties for $\mathrm{CdO}$ nanomorphotypes. Hence we strongly conclude that the dielectric relaxation time and grain interior resistance value obtained from impedance spectroscopic analysis also supports our claim of morphology-dependent electric polarization. As observed in Table $2, R_{g}$ value is highest for spherical morphology which decreases with increase in temperature in all morphologies.

The $\sigma_{g}$ value is lowest for spherical morphology which increases with increment of temperature in all cases. Similarly, the relaxation time $\tau_{g}$ is highest for spherical morphology which decreases with increment of temperature in all cases. All these electrical parameters of $\mathrm{CdO}$ nanomorphotypes obtained by fitting the experimental data to Eq. (4) also suggest that the dielectric constant of $\mathrm{CdO}$ morphotypes depends strongly on morphology. 


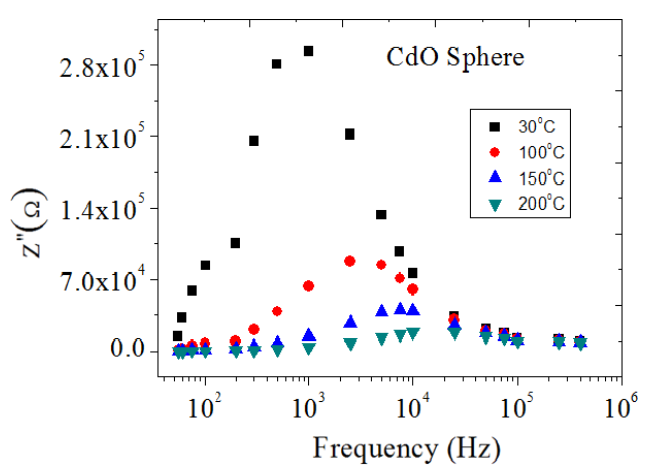

(a)

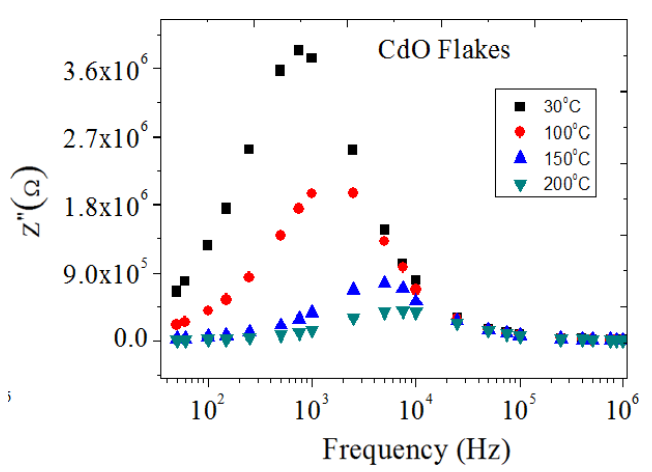

(b)

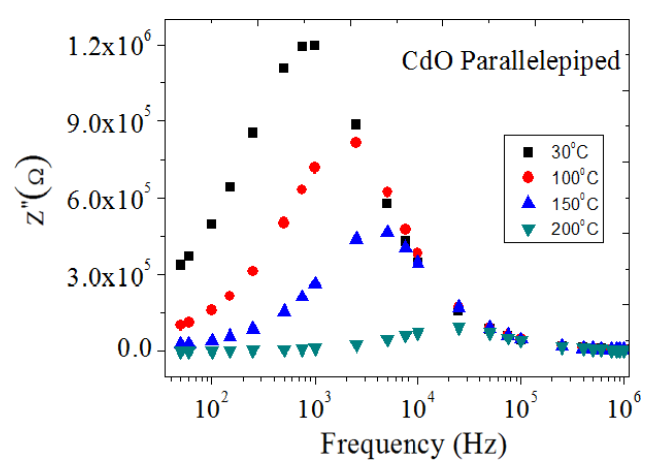

(c)

Fig. 5. $Z^{I I}$ versus frequency plot of CdO nanomorphotypes: (a) sphere, (b) flakes and (c) parallelepiped.

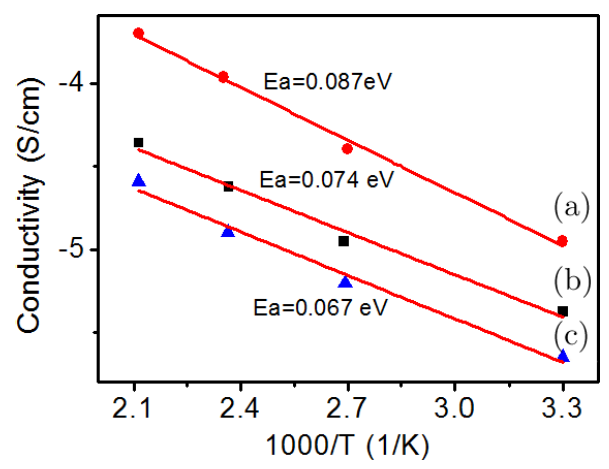

Fig. 6. Arrhenius plots of grain conductivity $\left(\sigma_{g}(\mathrm{~S} / \mathrm{cm})\right)$ data: (a) sphere, (b) flakes and (c) parallelepiped nanomorphotype.

\section{Conclusions}

In summary, we have evidently proved that the dielectric polarization and relaxation of $\mathrm{CdO}$ nanosphere, nanoflakes and nanoparallelepiped morphotypes are dependent on morphology. As the spherical nanomorphotypes has the maximum surface to volume ratio and surface area, the surface charge is maximum in it. Consequently the maximum surface/space charge polarization takes place in spherical nanomorphotype. The order of the dielectric constants obtained is: dielectric constant (d.c.) of nanosphere $>$ d.c. of nanoflakes $>$ d.c. of nanoparallelepiped. It is to be noted that the surface area of these morphotypes are also in the same order. The order of dielectric relaxation time and grain resistance values obtained from impedance spectroscopy are also in the same order. Therefore, the dielectric relaxation time and grain interior resistance value obtained from impedance spectroscopic analysis also strongly supports our claim of morphology-dependent electric polarization. The dielectric relaxation analysis suggests that the Cole-Cole relaxation mechanism is valid in the $\mathrm{CdO}$ morphotypes. The electrical study indicates that spherical $\mathrm{CdO}$ spherical nanomorphotype can be used as potential material candidate for new era of energy storage electronic devices.

\section{Acknowledgments}

The authors are thankful to the Royal Society of Chemistry for granting permission to reuse the XRD spectrum, BET spectrum and associated data of our paper published in the Journal of RSC Advances.

\section{References}

${ }^{1}$ Z.-M. Liao et al., Effect of surface states on electron transport in individual ZnO nanowires, Phys. Lett. A 376, 207 (2007).

${ }^{2} \mathrm{Z}$. Dang et al., Dielectric properties and morphologies of composite filled with whiskers and nanosized $\mathrm{ZnO}$, Mater. Res. Bull. 38, 499 (2003). 
${ }^{3} \mathrm{~S}$. Suresh, Studies on the dielectric properties of CdS nanoparticles, Appl. Nanosci. 4, 325 (2014).

${ }^{4}$ T. S. Velayutham et al., Theoretical and experimental approach on dielectric properties of $\mathrm{ZnO}$ nanoparticles and polyurethane/ $\mathrm{ZnO}$ nanocomposites, J. Appl. Phys. 112, 054106 (2012).

${ }^{5} \mathrm{M}$. A. El-Sayed, Some interesting properties of metals confined in time and nanometer space of different shapes, Acc. Chem. Res. 34, 257 (2001).

${ }^{6}$ S. W. Chung, J. Y. Yu and J. R. Heath, Silicon nanowire devices, Appl. Phys. Lett. 76, 2068 (2000).

${ }^{7} \mathrm{R}$. Cuscó et al., Raman scattering of cadmium oxide epilayers grown by metal-organic vapor phase epitaxy, J. Appl. Phys. 107, 063519 (2010).

${ }^{8} \mathrm{~S}$. Reddy et al., Synthesis of CdO nanoparticles and their modified carbon paste electrode for determination of dopamine and ascorbic acid by using cyclic voltammetry technique, Int. J. Electrochem. Sci. 5, 10 (2010).

${ }^{9}$ Y. W. Wang et al., Preparation and characterization of ordered semiconductor CdO nanowire arrays, J. Mater. Sci. Lett. 20, 1687 (2001).

${ }^{10} \mathrm{Y}$. Li and W. Shen, Morphology-dependent nanocatalysis on metal oxides, Sci. China Chem. 55, 2485 (2012).

${ }^{11}$ A. Dumbrava, V. Ciupina and G. Prodan, Dependence on grain size and morphology of zinc sulfide particles by the synthesis route, Rom. J. Phys. 50, 831 (2005).

${ }^{12}$ Landolt-Börnstein, Cadmium Oxide (CdO) Optical and Photoelectric Properties, Dielectric Constants, Plasmon Energy, Series Landolt-Börnstein - Group III Condensed Matter, 41B, 1-21 (1999).

${ }^{13} \mathrm{P}$. Lunkenheimer et al., Origin of apparent colossal dielectric constants, Phy. Rev. B 66, 052105 (2002).

${ }^{14} \mathrm{P}$. Thomas et al., Morphology dependent nanosecond and ultrafast optical power limiting of $\mathrm{CdO}$ nanomorphotypes, RSC Adv. 5, 35017 (2015).

${ }^{15}$ R. Tripathi, A. Kumar and T. P. Sinha, Dielectric properties of CdS nanoparticles synthesized by soft chemical route, Pramana $J$. Phys. 72, 969 (2009).

${ }^{16} \mathrm{~S}$. Mahboob, G. Prasad and G. S. Kumar, Impedance and a.c conductivity studies on $\mathrm{Ba}(\mathrm{Nd} 0.2 \mathrm{Ti} 0.2) \mathrm{O} 3$ ceramic prepared through conventional and microwave sintering route, Bull. Mater. Sci. 29, 347 (2006).

${ }^{17}$ Md. T. Rahman and C. V. Ramana, Impedance spectroscopic characterization of gadolinium substituted cobalt ferrite ceramics, J. Appl. Phys. 116, 164108 (2014).

${ }^{18}$ Y. P. Timalsina et al., Alternating current impedance spectroscopic analysis of biofunctionalized vertically-aligned silica nanospring surface for biosensor applications, J. Appl. Phys. 10, 014901 (2011).

${ }^{19} \mathrm{U}$. Kuhn and F. Lüty, Paraelectric heating and cooling with $\mathrm{OH}-$ dipoles in alkali halides, Solid State Commun. 3, 31 (1965).

${ }^{20} \mathrm{~J}$. Fan et al., Solution-growth and optoelectronic performance of $\mathrm{ZnO}: \mathrm{Cl} / \mathrm{TiO} 2$ and $\mathrm{ZnO}: \mathrm{Cl} / \mathrm{ZnxTiOy} / \mathrm{TiO} 2$ core-shell nanowires with tunable shell thickness, J. Phys. D: Appl. Phys. 45, 415301 (2012).

${ }^{21} \mathrm{~B}$. Cheng et al., Highly sensitive humidity sensor based on amorphous Al2O3 nanotubes, J. Mater. Chem. 21, 1907 (2011).
${ }^{22}$ D. C. Sinclair and A. R. West, Impedance and modulus spectroscopy of semiconducting $\mathrm{BaTiO} 3$ showing positive temperature coefficient of resistance, J. Appl. Phys. 66, 3850 (1989).

${ }^{23}$ K. S. Cole and R. H. Cole, Dispersion and absorption in dielectrics I. Alternating current, J. Chem. Phys. 9, 341 (1941).

${ }^{24}$ H.-M. Cheng et al., Formation of branched $\mathrm{ZnO}$ nanowires from solvothermal method and dye-sensitized solar cells applications, $J$. Phys. Chem. C, 112, 16359 (2008).

${ }^{25}$ L. Pandey et al., Equivalent circuit models for electronic ceramics, Bull. Mater. Sci. 18, 563, (1995).

${ }^{26}$ M. R. Shoar Abouzari et al., On the physical interpretation of constant phase elements, Solid State Ionics 180, 922 (2009).

${ }^{27}$ M. A. L. Nobre and S. Lanfredi, Grain boundary electrical characterization of $\mathrm{Zn}_{7} \mathrm{Sb}_{2} \mathrm{O}_{12}$ semiconducting ceramics: A negative temperature coefficient thermistor, J. Appl. Phys. 93, 5576 (2003).

${ }^{28}$ J. Liu et al., Large dielectric constant and Maxwell-Wanger relaxation in $\mathrm{Bi}_{2 / 3} \mathrm{Cu}_{3} \mathrm{Ti}_{4} \mathrm{O}_{12}$, Phys. Rev. B 70, 144106 (2004).

${ }^{29} \mathrm{~N}$. Kilınç et al., Structure and electrical properties of Mg-doped ZnO nanoparticles, Cryst. Res. Technol. 45, 529 (2010).

${ }^{30} \mathrm{~A}$. Tabib et al., Investigations on electrical conductivity and dielectric properties of $\mathrm{Na}$ doped $\mathrm{ZnO}$ synthesized from sol gel method, J. Alloys Compd. 622, 687 (2015).

${ }^{31}$ Ramna Tripathi et al., Dielectric relaxation of CdO nanoparticles, Appl Nanosci. 6, 175 (2016).

${ }^{32}$ International Programme on Chemical Safety and the European Commission IPCS 2004-2012, ICSC:0117.

${ }^{33}$ Center for disease control and prevention, CDC 24/7: National institute for Occupational safety and health (NIOSH).

${ }^{34}$ H. A. Haus and J. R. Melcher, Electromagnetic Fields and Energy, Englewood Cliffs, NJ: Prentice-Hall (1989).

${ }^{35} \mathrm{P}$. Mechant and C. Elbarum, Anisotropic conductivity in plastically deformed ZnS, Solid State Commun. 26, 73 (1978).

${ }^{36} \mathrm{~S}$. Suresh and C. Arunseshan, Dielectric properties of cadmium selenide (CdSe) nanoparticles synthesized by solvothermal method, Appl. Nanosci. 4, 179 (2014).

${ }^{37}$ E. S. P. Leong et al., Effect of surface morphology on the optical properties in metal_dielectric_metal thin film systems, Appl. Mater. Interf. 3, 1148 (2011).

${ }^{38} \mathrm{~J}$. Li et al., The effect of nanoparticle surfactant polarization on trapping depth of vegetable insulating oil-based nanofluids, Phys. Lett. A 380, 604 (2016).

${ }^{39} \mathrm{Z}$. Yuanxiang et al., Morphology effects on space charge characteristics of low density polyethylene, Jpn. J. Appl. Phys. 50, 017101 (2011).

${ }^{40}$ T. V. S. L. Satyavani et al., Effect of particle size on dc conductivity, activation energy and diffusion coefficient of lithium iron phosphate in Li-ion cells, Eng. Sci. TechnolInt. J. 19(1), 40 (2016).

${ }^{41}$ M. C. J. M. Vissenberg and M. Matters, Theory of the field-effect mobility in amorphous organic transistors, Phys. Rev. B 57(20), 12964 (1998).

${ }^{42}$ I. G. Austin and N. F. Mott, Polarons in crystalline and noncrystalline materials, Adv. Phys. 18(71), 41 (1969).

${ }^{43} \mathrm{M}$. Wimmer et al., Role of activation energy in resistance drift of amorphous phase change materials, Front. Phys. 2, 1 (2014). 